\title{
Controlling the Organization of PEDOT:PSS on Cellulose Structures
}

Belaineh, Dagmawi; Andreasen, Jens W.; Palisaitis, Justinas; Malti, Abdellah; Håkansson, Karl; Wågberg, Lars; Crispin, Xavier; Engquist, Isak; Berggren, Magnus

\section{Published in:}

ACS Applied Polymer Materials

Link to article, DOI:

10.1021/acsapm.9b00444

Publication date:

2019

Document Version

Peer reviewed version

Link back to DTU Orbit

Citation (APA):

Belaineh, D., Andreasen, J. W., Palisaitis, J., Malti, A., Håkansson, K., Wågberg, L., Crispin, X., Engquist, I., \& Berggren, M. (2019). Controlling the Organization of PEDOT:PSS on Cellulose Structures. ACS Applied Polymer Materials, 1(9), 2342-2351. https://doi.org/10.1021/acsapm.9b00444

\section{General rights}

Copyright and moral rights for the publications made accessible in the public portal are retained by the authors and/or other copyright owners and it is a condition of accessing publications that users recognise and abide by the legal requirements associated with these rights.

- Users may download and print one copy of any publication from the public portal for the purpose of private study or research.

- You may not further distribute the material or use it for any profit-making activity or commercial gain

- You may freely distribute the URL identifying the publication in the public portal 


\section{Controlling the Organization of PEDOT:PSS on Cellulose Structures}

Dagmawi Belaineh ${ }^{*+1}$, Jens W. Andreasen ${ }^{2}$, Justinas Palisaitis ${ }^{3}$, Sapiens Malti ${ }^{4}$, Karl

Håkansson $^{5}$, Lars Wågberg ${ }^{4}$, Xavier Crispin ${ }^{1}$, Isak Engquist* ${ }^{*}$, Magnus Berggren ${ }^{1}$

1. Laboratory of Organic Electronics

Department of Science and Technology

Linköping University

60174 Norrköping

Sweden

†Present Address

RISE Acreo

Research Institutes of Sweden

60117 Norrköping

Sweden 
2. Department of Energy Conversion and Storage

Technical University of Denmark

4000 Roskilde

Denmark

3. Department of Physics, Chemistry, and Biology

Linköping University

58183 Linköping

Sweden

4. Fibre and Polymer Technology, and Wallenberg Wood Science Center

KTH Royal Institute of Technology

10044 Stockholm

Sweden

5. RISE Bioeconomy

Research Institutes of Sweden

11486 Stockholm

Sweden 
Keywords: nanocomposites, biomaterials, PEDOT, nanotechnology, energy materials, cellulose

Composites of biopolymers and conducting polymers are emerging as promising candidates for a green technological future and are actively being explored in various applications, such as in energy storage, bioelectronics, and thermoelectrics. While the device characteristics of these composites have been actively investigated, there is limited knowledge concerning the fundamental intra-component interactions and the modes of molecular structuring. Here, using cellulose and poly(3,4-ethylenedioxythiophene):poly(styrenesulfonate) (PEDOT: PSS) it is shown that the chemical and structural make-up of the surfaces of the composite components are critical factors that determine the materials organization at relevant dimensions. AFM, TEM and GIWAXS measurements show that when mixed with cellulose nano-fibrils, PEDOT:PSS organizes into continuous nano-sized bead-like structures with an average diameter of $13 \mathrm{~nm}$ on the nano-fibrils. In contrast, when PEDOT:PSS is blended with molecular cellulose, a phasesegregated conducting network morphology is reached, with a distinctly relatively lower electric conductivity. These results provide insight into the mechanisms of PEDOT:PSS crystallization and may have significant implications for the design of conducting-biopolymer composites for a vast array of applications.

At this time of rapid human development, environmentally friendly means of addressing the world's increasing technological demands are needed more than ever. Composite systems of electronically active polymers and renewable biomaterials are seen as one of the key solutions in this drive towards a green and clean planet. ${ }^{1-5}$ Conducting polymers are frequently blended with biopolymers for an array of novel applications where the biopolymers provide hierarchical 
flexibility, ${ }^{6}$ improve performance, ${ }^{7}$ mechanical stability, ${ }^{8}$ processability, ${ }^{9}$ and biocompatibility ${ }^{10}$ to the pure conducting polymer, leading to powerful synergistic performance and device functions. For example, the production of stand-alone bulk supercapacitor electrodes has recently been enabled via a amalgamated system including the conducting polymer poly(3,4-ethylenedioxythiophene):poly(styrene-sulfonate) (PEDOT:PSS, Figure 1a) and the mechanically strong cellulose nanofibril (CNF, Figure 1c). ${ }^{8}$ The resulting conducting paper, called power paper, is flexible, scalable and exhibits excellent mechanical and electrical properties including high mixed ionic and electronic conductivity. The addition of cellulose to the PEDOT:PSS enables us to manufacture sustainable, thick and bulky supercapacitor electrodes, which is not possible to achieve using PEDOT:PSS only. However, notwithstanding the promising properties of such systems, there is a lack of clear structural paradigms guiding the selection of compatible conducting polymers and biopolymers.

a)

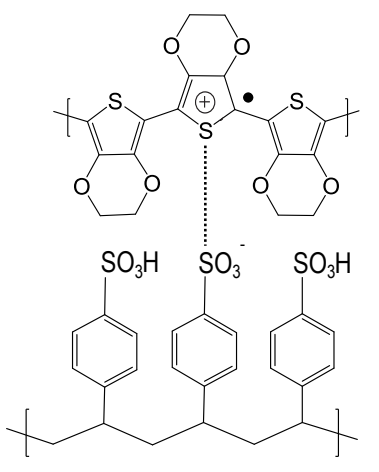

c)

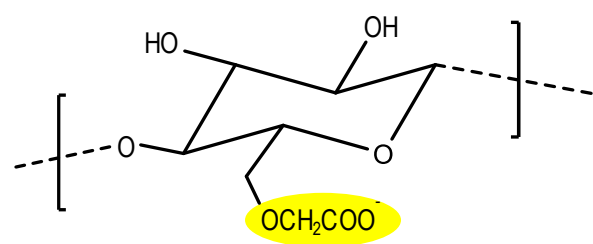

PEDOT

PSS d)

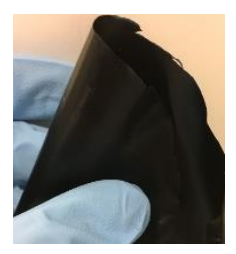

b)

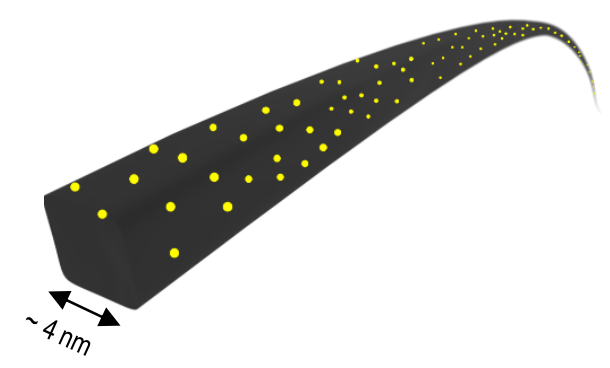


Figure 1. a) Chemical structure of PEDOT:PSS b) schematic of CNF with carboxymethyl groups (yellow) c) chemical structure of carboxymethylated cellulose (CMC) with degree of substitution (DS) 1. d) power paper (CNF-PEDOT:PSS conducting paper) e) CMC-PEDOT:PSS conducting paper.

Wood is the most readily available and environmentally friendly biomaterial. ${ }^{1}$ Wood has a hierarchical structure which makes it an essential source of an array of biomaterials of different sizes and chemical compositions. One of the main components of wood, cellulose, has a long list of commendable attributes and is commercially available in a vast array of different structural forms. ${ }^{11,12}$ The anisotropic micron to millimeter sized fibers that constitute a regular sheet of paper can be mechanically and/or chemically treated to give highly anisotropic cellulose nanofibril (CNF) ${ }^{13}$ CNF (Fig. 1c) is being investigated in several applications within bioactivity, ${ }^{14}$ gel formation, ${ }^{15}$ transparent substrates, ${ }^{16}$ and composite structures. ${ }^{17} \mathrm{CNF}$ is often prepared with some of its hydroxyl groups substituted with functional ionic groups such as carboxymethyl groups to facilitate dispersibility in water. ${ }^{18}$ The degree of substitution (DS-value) quantifies the number of hydroxyl groups per monomer unit that have been substituted with a charged group. Typically, CNF will have a DS between 0.05 and 0.35 . It should be noted that, as the substituted charges reside only on the surface of the CNF, the surface charge density of the fibril is high. For a DS of 0.35 the average distance between adjacent charged groups is $\sim 0.7 \mathrm{~nm}$. Beyond a DS of 0.4 the glucan chain becomes so highly charged that it will dissolve into the polyelectrolyte form, i.e. carboxymethyl cellulose (CMC, Figure 1b). ${ }^{19}$ Owing to its physiological inertness and high water solubility, polymeric $\mathrm{CMC}$ has been in use for a long time as a viscosity regulating food additive and, recently, $\mathrm{CMC}$ has also been actively investigated as a safe binder material in state-of-the-art batteries and supercapacitors. ${ }^{20}$ 
The stability and high mixed electronic-ionic conductivity of PEDOT:PSS (Fig. 1a) has made it the commercially most successful conducting polymer, ${ }^{21,22}$ even though several high-performing alternatives can be found in the various families of conjugated polymers. ${ }^{23,24}$ The polyanion PSS neutralizes and dopes the charged PEDOT, while excess PSSH in the system makes the whole material dispersible in water and provides easy processing. ${ }^{25} \mathrm{PEDOT}$ :PSS's mixed ionic/electronic charge transport capability has opened up an array of applications, such as organic electrochemical transistors and organic electronic ion pumps. ${ }^{26}$ Recently, PEDOT:PSS is being intensively investigated for energy generation and storage applications, such as in fuel cells, thermoelectrics and supercapacitors. ${ }^{8,27-30}$

The morphology of PEDOT:PSS has actively been studied with a range of techniques. ${ }^{31-33} \mathrm{In}$ solution state, PEDOT:PSS is found as a nano-suspension with $\sim 30 \mathrm{~nm}$ size particles and 500 $600 \mathrm{~nm}$ size aggregates of the particles. Individual particles are composed of an inner core of PEDOT-rich region surrounded by a soft PSS-rich region. ${ }^{34-36}$ On the other hand, dried and thinfilms of PEDOT:PSS are depicted as segregated PEDOT-rich and PSS-rich regions. The PEDOTrich regions are composed of crystalline grains of a few nanometers size, organized into clusters of tens to a hundred nanometers depending on the details of the sample preparation. ${ }^{31,37,38}$ It has been reported that so-called secondary dopants such as dimethyl-sulfoxide (DMSO), ethylene glycol, and methanol have an impact on the phase separation and organization of PEDOT and PSS. This phase separation results in a highly interconnected PEDOT network with stronger interchain coupling of the PEDOT (PEDOT $\pi$-stacking), thereby improving the electronic conductivity significantly. ${ }^{39-42}$ The effect of the substrate on PEDOT:PSS morphology is largely unexplored but recent theoretical studies suggest that the interaction of PEDOT:PSS with the substrate is critical for its organization and to promote swift charge transport. ${ }^{43}$ 
PEDOT:PSS-based self-standing conducting paper can be made from either CMC or CNF for energy storage applications (Figure 1d and e). Both kinds of conducting papers are mechanically resilient to continuous folding and rolling. However, the fibril-based papers show improved electrical device characteristics as compared to their polymeric counterparts, indicating either a different structuring, or differences in the interaction between PEDOT:PSS and cellulose, or both. It has also been shown that the level of adsorption of PEDOT:PSS on cellulose is dependent on the $\mathrm{pH}$ of the system. ${ }^{44}$ PEDOT:PSS shows maximum adsorption on cellulose at its natural $\mathrm{pH}$ levels, which are in the range of 2 due to the low pKa of the sulfonic acid groups. At such low $\mathrm{pH}$ levels, the charged groups of the cellulose are protonated, and the charged fibrils become neutral. Hence, the electrostatic repulsion between the cellulose and the negatively charged sulfonate groups of the PEDOT:PSS is at its minimum and Van der Waals and dipolar interactions dominate. This means that at low $\mathrm{pH}$ levels the interactions of cellulose with PEDOT:PSS are not primarily dictated by the DS of the cellulose. The superior device properties observed in the fibrillated samples seems to suggest that the geometrical structure of the cellulose plays a critical role in PEDOT:PSS organization.

Even though earlier investigations have to some degree addressed the interactions between the constituents of a composite and the structural organization, there is only limited knowledge of the hierarchical arrangement of composites from the molecular to the micrometer size scale. ${ }^{45-48}$ Therefore, despite the generic interest in blend systems composed of conducting polymers and biomaterials, there is a lack of clear understanding of the structural organization of these composites and its impact on device performance. This is partly due to the complexity of the composite, making sample preparation, measurement and data analysis a challenging task. 
Here, by using two structurally different cellulose systems in a composite system with PEDOT:PSS, we show that the PEDOT:PSS organizes itself in nanometer-sized bead-like structures on top of CNF nanofibers with predominantly pi-stacked PEDOT, whereas CMC brings about an organization which is dominated by phase separation and stronger lamellar stacking of PEDOT. The structural variation is also consistent with a distinct improvement in electronic conductivity in the fibril-based systems. We use Wide Angle X-ray Scattering (WAXS) for crystallographic and textural investigations and direct measurements with Atomic Force Microscopy (AFM) and Transmission Electron Microscopy (TEM), to show the distinct morphology and crystallographic organization of the conducting polymer in the different cellulosic systems. The necessity of understanding and tailoring the structure and chemistry of the constituents of bio-composites for more efficient devices is emphasized.

\section{RESULTS AND DISCUSSION}

The conductivity of the power-papers was studied with four-probe electrical measurements. Figure 2a shows the variation of conductivity with the degree of carboxymethylation (DS) of the cellulose. Fibrils (CNF) correspond to DS values below 0.4 while above this value the cellulose is found in the form of a polymeric CMC. We observe two distinct regimes with respect to the amount of carboxymethyl charge on the cellulose. In the low-charge, fibrillated zone, the conductivity is higher with values in the range of $400 \mathrm{~S} / \mathrm{cm}$. At higher charge contents, where the cellulose is polymeric and completely soluble in water, the conductivity is lower $(150 \mathrm{~S} / \mathrm{cm})$. The fact that the conductivity values stay relatively constant within these two regimes suggests that the PEDOT organization is not varying markedly in the respective regimes but that there is distinct conductivity level difference between the two material systems. 
By studying previous literature, which reports the effect of adding excess of PSS to PEDOT:PSS, we can compare the role of the non-conducting fibrils in the conductivity of the paper with the role of a non-conducting polymer (PSS). ${ }^{49}, 50$ The amount of the electronically conducting component, PEDOT, in the power paper is only around $20 \%$ by weight, and the remaining component is the non-conducting cellulose together with PSS. A renormalized conductivity with respect to the weight of PEDOT gives a value of about $2000 \mathrm{~S} / \mathrm{cm}$ for the PEDOT-component in the fibril-based paper. This value is more than half of the renormalized conductivity of PEDOT:PSS which is 3500 S/cm at 1:2.5 PEDOT:PSS ratio. ${ }^{51}$ However, if we replace the amount of cellulose in power paper by PSS, the renormalized conductivity is then less than $700 \mathrm{~S} / \mathrm{cm}$. It is then clear that the addition of excess PSS has a more detrimental effect on the conductivity than the addition of fibrils to PEDOT:PSS. It can also be seen that the replacement of CNF by CMC in power paper also has a similar effect as adding excess PSS.
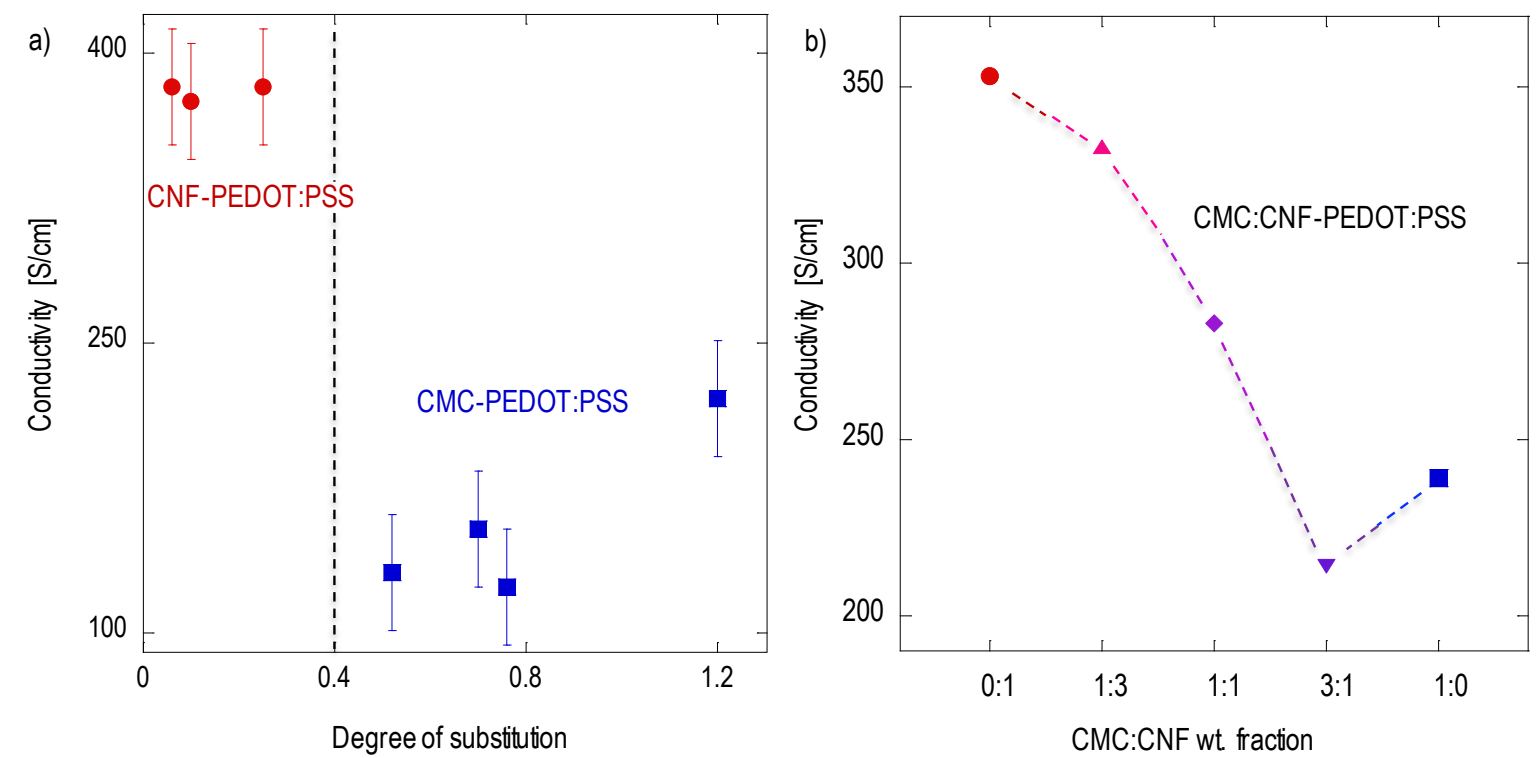

Figure 2. a) Conductivity of CNF-PEDOT:PSS and CMC-PEDOT:PSS conducting papers b) Conductivity of conducting paper at different CMC:CNF weight fractions. 
We further investigated the role of the cellulose in determining the conductivity of the PEDOT by mixing CNF and CMC with PEDOT:PSS to prepare the conducting paper. Figure 2b display the electrical results from experiments where the addition of CMC to the CNF brings about a decrease in the conductivity of the conducting paper. The conductivity decreases until the ratio of CNF:CMC is 1:3 and then increases slightly for the pure CMC-PEDOT:PSS sample, but still at a level much lower than for pure CNF-PEDOT:PSS. Hence, an addition of a small amount of CNF to a $\mathrm{CMC}$ solution, or vice versa, leads to a significant drop of conductivity. This result suggests that the pure $\mathrm{CNF}$ and $\mathrm{CMC}$ blends promote totally different kinds of PEDOT organization. An addition of a small amount of CNF to CMC-PEDOT:PSS results in disorder in the charge transport pathway and leads to a decrease of conductivity. However, with more CNF in the system the CNFgoverned organization of PEDOT takes precedence. Since this organization leads to higher conductivity, the conductivity of the blend system increases until it reaches the maximum conductivity for blends made with pure CNF-PEDOT:PSS.

Charge transport in PEDOT:PSS films is mainly governed by the ease of charge transfer between clusters of PEDOT:PSS. ${ }^{31,52,53}$ The PEDOT:PSS film is understood to be a phase-separated mix of PEDOT-rich clusters surrounded by PSS-rich lamellae. ${ }^{54}$ Nardes et al report that a thermally activated charge transport dominates at room temperature and that the anisotropy introduced by phase separation leads to large variations in the conductivity values in the lateral and perpendicular directions. ${ }^{31}$ The differences in conductivity between the CNF-based and the CMC-based samples indicate that there is a marked difference in the charge transport mechanism and, consequently, in the organization of the PEDOT of the two blend systems. In what follows, AFM and WAXS measurements show that there is a more pronounced $\pi$-stacking and improved long range domain organization in the CNF samples, which are both known to lead to higher conductivity. ${ }^{37,55}$ 
Even though AFM is a surface sensitive technique which typically cannot give details of bulk morphology, it is a powerful method to clarify the influence of interactions between the components of a composite system, and, in combination with other techniques, can provide critical information of the phase separation and organization of the material. The topographic AFM image of the pure CNF in Figure 3a shows well dispersed, high aspect-ratio fibrils. Since the fibrils are smaller than the lateral resolution limit, of around $5-10 \mathrm{~nm}$ dictated by the tapping-mode AFM tip, the vertical profile was used to measure the dimension of the fibrils. It was determined that the fibrils have diameters in the range of 3-5 $\mathrm{nm}$. When the CNF-PEDOT:PSS composite is examined (Figure 3b), we see evidence of bead-like PEDOT:PSS structures aligned along the fibrils. The zoom-in image of the center part (Figure 3g) shows in detail how spherical clusters of PEDOT:PSS form a continuous bead-path. To extract the size of the beads, the length of a series of beads on a single fibril was averaged. On an average, the beads are $13 \pm 1 \mathrm{~nm}$ in diameter. However, we observe a few outliers with diameters as large as $25 \mathrm{~nm}$. These nano-beads have a size which is in agreement with dynamic light scattering (DLS) measurements of PEDOT:PSS particles in the solution state..$^{34,35}$

To investigate the nature of the beads, the phase image of the scans was studied. From previous phase image studies of PEDOT:PSS it is known that PSS, which adsorbs water and is a relatively softer material than PEDOT, has a lower phase angle and appears thus as relatively darker. ${ }^{56}$ Regions which are PEDOT-rich are represented by brighter areas in the image. In Figure $3 e$ it is seen that the individual beads have varying darkness suggesting that the PSS coverage is nonuniform. Based on models of PEDOT:PSS, which predict a PSS-rich outer cladding and a PEDOTrich core, we suggest that the observed beads are PEDOT:PSS particles with an irregular PSS-rich outer region and an inner PEDOT-rich region. 

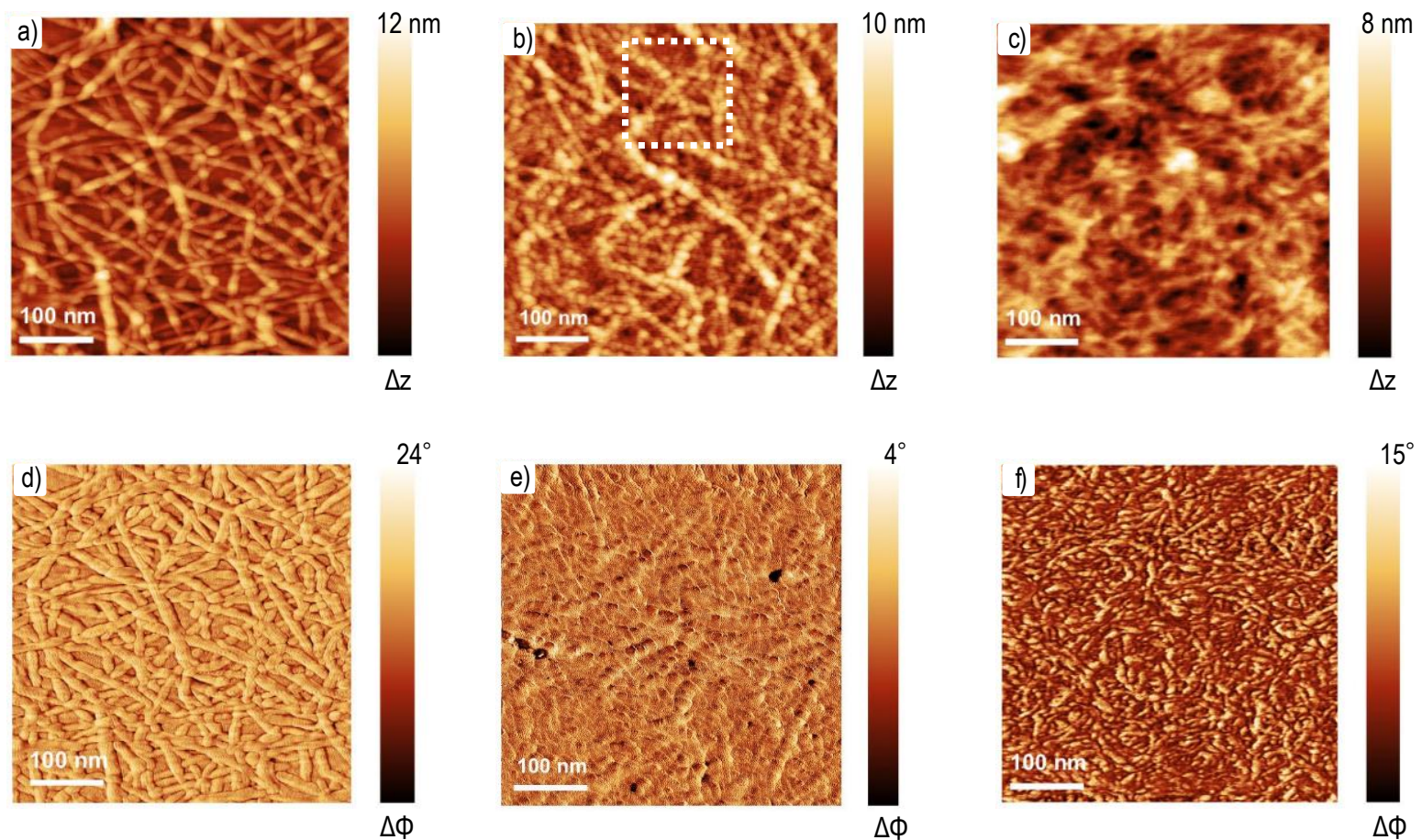

$15^{\circ}$

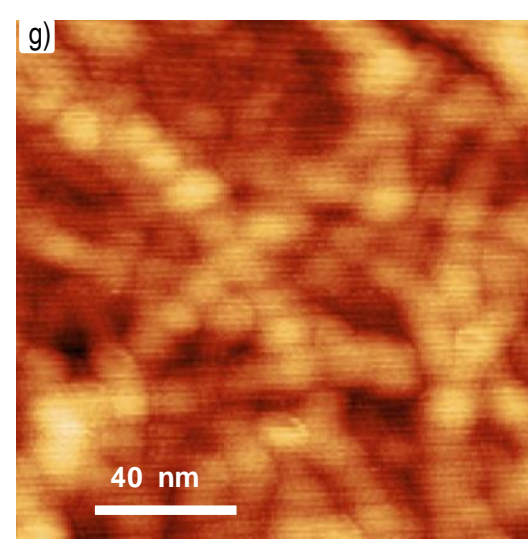

$10 \mathrm{~nm}$

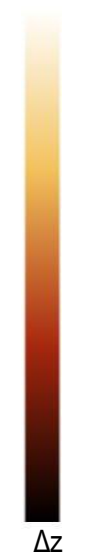

Figure 3. AFM topography and phase images of a) and d) pure CNF, b) and e) PEDOT:PSS on CNF, c) and f) CMC-PEDOT:PSS, respectively. g) a zoom-in image of the area marked with a dotted rectangle in b) (PEDOT:PSS on CNF).

Figure $3 \mathrm{c}$ displays the topography of CMC-PEDOT:PSS. The surface is a mix of a few globular structures with sizes in the range of 40-60 nm and some elongated structures with lengths $75-95$ $\mathrm{nm}$. The phase image (Figure 3f) that was simultaneously taken shows a phase separated brighter 
phase which is a mix of globular and elongated structures. As in the previous phase image, the bright parts depict the PEDOT-rich regions. The CMC, similar to PSS, is hygroscopic and is expected to swell at ambient conditions, hence becoming softer than its surrounding and thus have a relatively lower phase (dark region). In support of this interpretation, the phase difference observed in a CMC-PSS mix is minimal (Supporting Information, Figure S1d) indicating that they indeed have similar mechanical properties at ambient conditions. The phase image in Figure $3 \mathrm{f}$ shows a reduction in the interconnectivity of the PEDOT-rich regions as compared to a pure PEDOT:PSS film (Supporting Information, Figure S1c). Therefore, it appears that CMC brings about a disruption in the phase segregation process. Even though the PSS and CMC segregate, giving rise to PEDOT-rich regions, the PEDOT-rich regions are not organized into interconnected structures to the extent as found in pure PEDOT:PSS. This reduction in the interconnectivity of the PEDOT-rich regions is likely the cause for the higher drop of the conductivity in the CMCPEDOT:PSS blends.

To establish the form of PEDOT organization, WAXS measurements in transmission (with the X-ray beam impinging perpendicular to the film surface) and in Grazing Incidence (GIWAXS, with the X-ray beam nearly parallel to the film surface) were performed. WAXS is a suitable technique for probing the structural ordering of polymers from a few to several hundred nanometers. ${ }^{57}$ The transmission and GIWAXS modes can be used complementarily to derive a semi 3-dimensional representation of the investigated samples. 

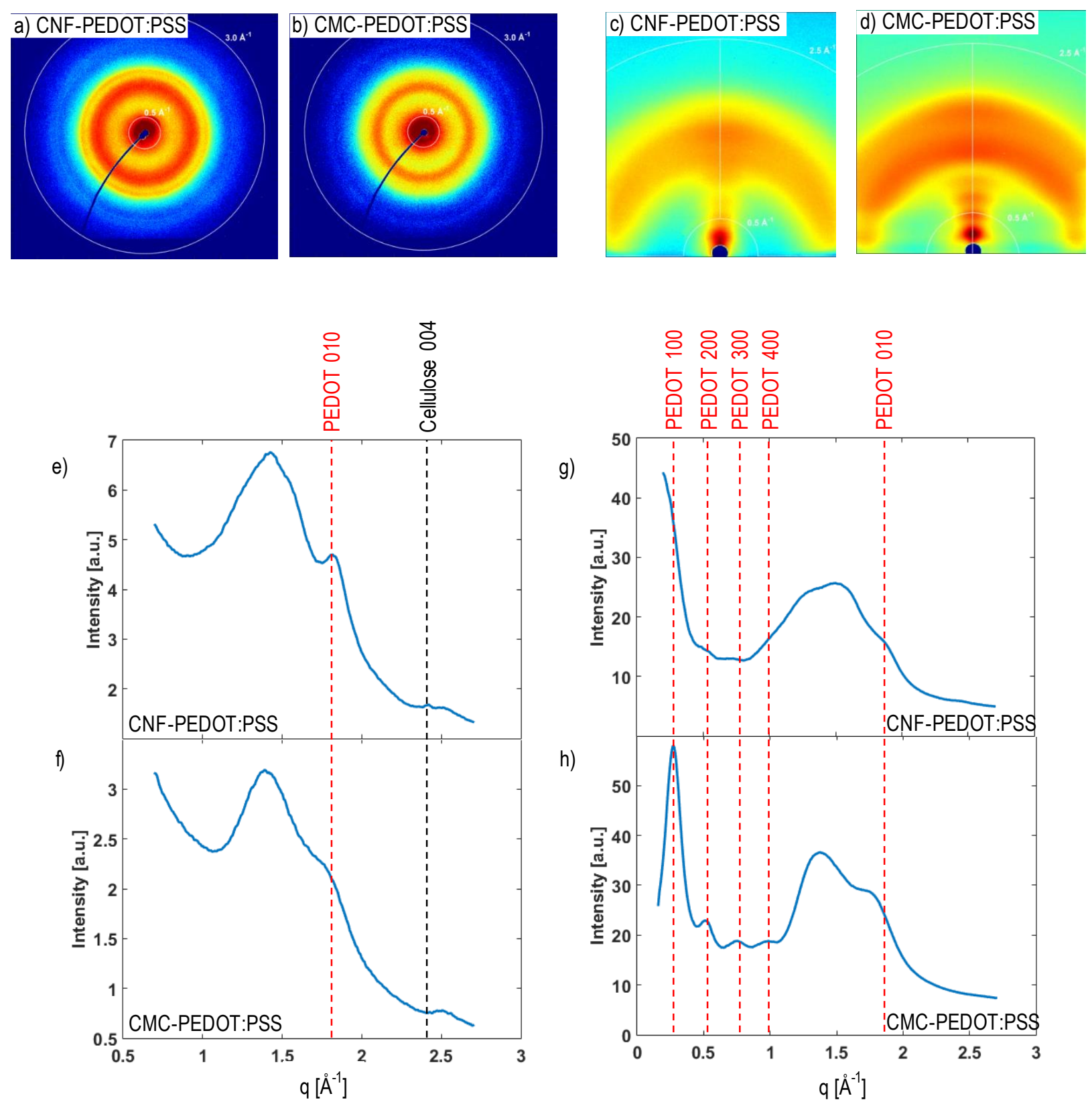

Figure 4. WAXS in transmission incidence for conducting paper made with PEDOT:PSS and a) $\mathrm{CNF}$ b) $\mathrm{CMC}$ and GIWAXS in parallel incidence c) CNF and d) CMC. The corresponding azimuthal integration for transmission incidence for e) $\mathrm{CNF}$ f) $\mathrm{CMC}$ and parallel incidence g) $\mathrm{CNF}$ and h) CMC. 
Figure 4 shows WAXS data of free-standing films made from PEDOT:PSS mixed with CNF or CMC. It was noted that the WAXS data for CNF samples of different DS were qualitatively similar to one another. The same observation was made for CMC samples of different DS. Therefore, patterns collected in the transmission and parallel configuration for the samples DS 0.12 and DS 1.2 are shown as representative examples of fibrillated and soluble samples, respectively. WAXS data for the other charge groups can be found in the supplementary information. For ease of comparison, the WAXS pattern data of all samples has been azimuthally integrated and the intensity plotted with respect to the length of the scattering vector, $|q|=4 \cdot \pi \cdot \sin \theta / \lambda$, where $2 \theta$ is the scattering angle and $\lambda$ is the X-ray wavelength.

From the transmission measurements of the fibrillated samples (Figure 4a and 4e) we observe that PEDOT on CNF has $\pi-\pi$ ordering as can be seen from the PEDOT 010 peak at $q=1.80 \AA^{-1}$, corresponding to a real space distance of $3.5 \AA$, as has been reported previously. ${ }^{23,51}$ The collected diffraction patterns (Figure 4a) shows continuous rings indicating PEDOT 010 stacks randomly oriented with respect to the film surface normal (fiber symmetry), i.e. edge-on orientation (Supporting Figure S4 illustrates the different types of PEDOT packing). The cellulose 012 and 020 rings observed at scattering vector $q=1.41 \AA^{-1}$ and $q=1.51 \AA^{-1}$, respectively, demonstrate that the fibrils are randomly oriented too. The grazing incidence pattern (Figure $4 \mathrm{c}$ and $4 \mathrm{~g}$ ), on the other hand, gives information of the crystal packing along the substrate normal, and shows the additional population of a predominantly $\pi-\pi$ stacked PEDOT in face-on orientation as indicated by the bright 010 arc at $90^{\circ}$ azimuthal angle (on the surface normal), with weak $\pi-\pi$ ordering at other angles. No significant lamellar order as can be seen in the weak scattering at small angles corresponding to the expected position of $h 00$ PEDOT peaks. Observing the AFM (Figure 3), it is reasonable to assume that the CNF takes the primary role in the organization of the PEDOT due 
to the large surface area it provides. In summary, the free-standing power paper is composed of two populations of PEDOT covering the CNF with $\pi$ - $\pi$ stacking occurring both face-on and edgeon with respect to the substrate. Such an organization is consistent with both AFM and WAXS data.

Molecular dynamic simulations (Supporting Figure S5) by Mehandzhiyski et al ${ }^{58}$ of different simplified systems containing only PEDOT:PSS, one fibril and water, show that in certain systems PEDOT arranges itself in bead-like aggregates with a lower density region in-between the beads. The similarity to the observed bead-like structures in Figure 3 is noteworthy. In such systems the orientation of the PEDOT is predominantly face-on on top of cellulose fibrils. Furthermore, simulated diffraction patterns of the same system computed by the same authors show distinct PEDOT $\pi-\pi$ stacking peaks. Significantly, the simulated diffraction patterns did not indicate any lamellar structures of PEDOT and, taken as a whole, the simulations are thus strongly corroborating the experimental AFM and WAXS data reported here.

The transmission WAXS data of the CMC-PEDOT:PSS samples (Fig. 4b and 4f) show a very weak PEDOT 010 signal, which seems to indicate the absence of $\pi-\pi$ edge-on ordering of PEDOT. Contrary to these observations, in the GIWAXS measurements of CMC-PEDOT:PSS paper we detect very strong lamellar peaks which indicate the presence of edge-on PEDOT crystals. In addition, similar to the fibril containing samples, face-on stacked PEDOT is indicated by the arc at $90^{\circ}$ azimuthal angle. Hence, the CMC-PEDOT:PSS samples have a mix of edge-on and faceon oriented PEDOT. We note that there are multiple factors influencing the organization of the PEDOT. Since this is a truly bulk material (sample thickness is several tens of microns) it is possible that the formation of PEDOT crystallites in the bulk is markedly different to the PEDOT crystallite formation at the surface in contact with the petri-dish during drying. Based on 
computational studies, Franco-Gonzalez et al. report that amorphous substrates, such as the glass petri-dish substrate used in this experiment, promote a predominantly edge-on morphology in wet state and a mixed edge-on/face-on morphology in dry state. ${ }^{43}$ Even though these theoretical models were done for PEDOT-Tosylate and do not contain cellulose, PSS, DMSO and glycerol, which are present in our films, these studies are noteworthy because they address the importance of the supporting substrate and the water content. Due to the wetness present due to the glycerol used as a plasticizer and to the hygroscopic nature of PSS and cellulose we can conjecture a mixed organization with a possible preference for edge-on orientation. It is possible that both edge-on and face-on orientations are present both at the surface and bulk of the free-standing paper. However, it is not clear which specific phenomenon dictates the PEDOT structuring in the bulk of the CMC-PEDOT:PSS samples.

To investigate the crystalline organization of PEDOT in the mixed systems we performed highresolution transmission electron microscopy (HRTEM). Figure 5 shows overview TEM images of CNF-PEDOT:PSS solution cast on a TEM copper grid where a few fibrils on the edge of the film can be observed. Enhanced magnification image of one of the fibrils (Fig 5a inset) shows that surrounding it, there is a stack of crystals with a stacking distance $\sim 4 \AA$. This coincides well with the $\sim 3.5 \AA \pi$-stacking distance of PEDOT crystals and suggests the observation of PEDOT crystals stacked face-on on the surface of the fibril. In addition, there is a thin, amorphous, nonuniform covering layer on top of the crystal stacks which we expect to be a PSS outer layer that is bound to the PEDOT. Furthermore, we observe that these crystalline stacks are not perfectly continuous on top of the fibril indicating that the PEDOT crystals may be organized on the surface of the fibrils in the nano-bead structures observed in the AFM images. 
Figure $5 \mathrm{~b}$ shows highly organized crystalline lattices with inter-lattice distance of $\sim 3.7 \AA$ of CNF-PEDOT:PSS solution cast on a TEM copper grid. The large crystalline structures are predominantly oval in shape and their size ranges from $\sim 5 \mathrm{~nm}$ up to $\sim 25 \mathrm{~nm}$ in width. On the other hand, neither the control pure PEDOT:PSS samples nor the CMC-PEDOT:PSS samples show any similar crystalline structures (Supplementary Figure 6). The size of most of the crystalline structures in Figure $5 \mathrm{~b}$ is comparable to the size range observed in the AFM images of the PEDOT structures on top of the fibrils. However, it is not possible to identify the fibril in these images. Therefore, we suggest that these are PEDOT crystallites which were formed in the following steps: 1) PEDOT aggregates on the CNF surface in the wet state 2) the PEDOT aggregate detaches from the fibril with its structure intact in the wet state and finally 3) forms highly organized crystallites when dried.
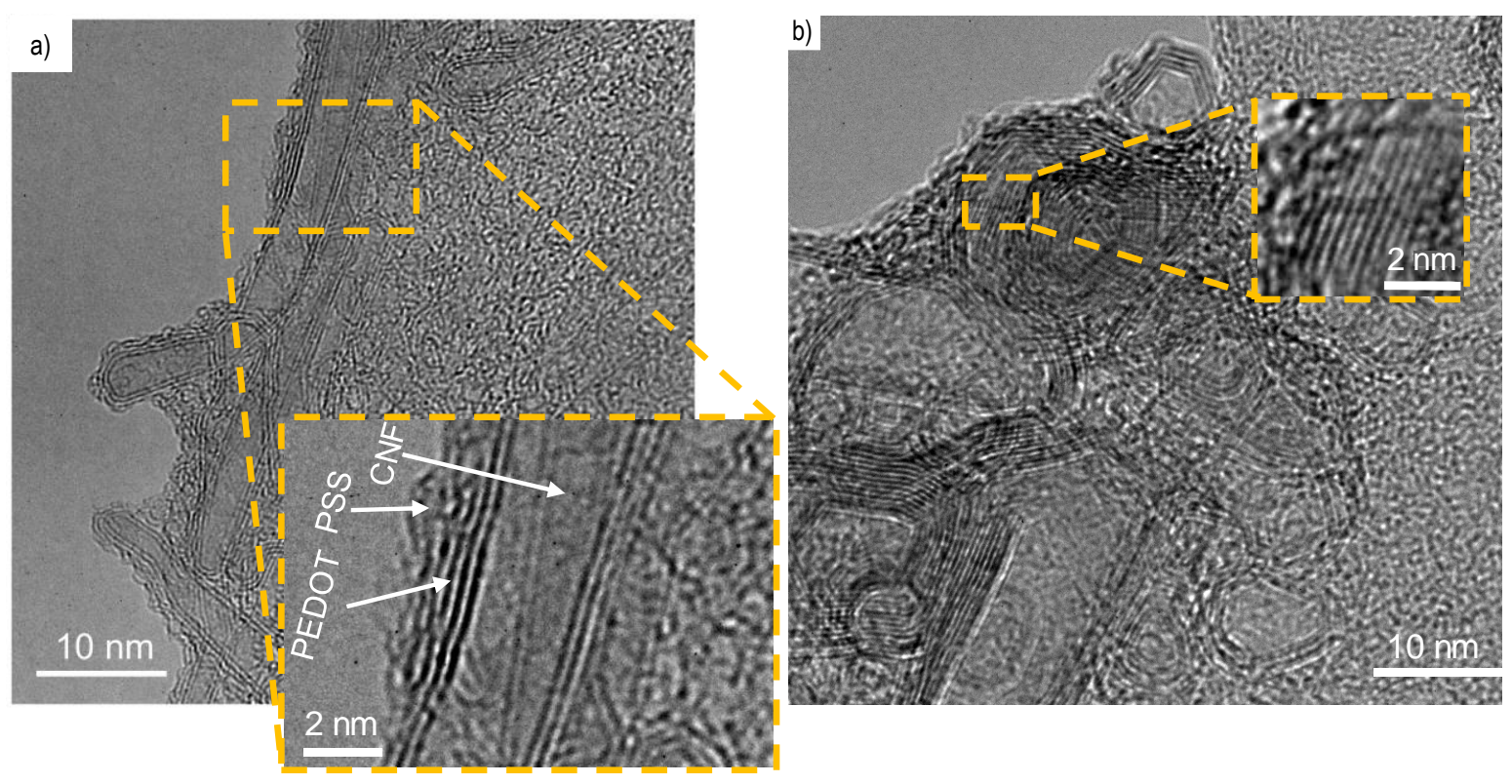

Figure 5. TEM image of a)PEDOT:PSS on the surface of CNF and b) highly crystalline PEDOT:PSS segregated from CNF surface. Insets show magnified images of the selected areas. 
Understanding the interactions between the constituents of a composite system and their impact on performance parameters are key steps towards building efficient devices. With the combined knowledge obtained from the different techniques it is possible to construct a reasonable model of the PEDOT organization in the presence of CNF and CMC.

For the CNF-PEDOT:PSS samples, the AFM scans show that the fibrils predominantly lie in the plane of the substrate, as expected. In addition, the AFM scans show that the PEDOT organizes itself in $13 \mathrm{~nm}$ bead-like structures, assembling itself around the fibrils, pointing to a strong ordering drive in the system. The GIWAXS transmission incidence data shows a symmetric $\pi$ stacking indicating that there are stacks of PEDOT in edge-on orientation with respect to the substrate surface. The parallel incidence GIWAXS shows a strong $\pi$-stacking peak at the $0^{\circ}$ azimuthal angle indicating a face-on PEDOT stacking with respect to the substrate plane as well. The strong $\pi$-stacking and the weak lamellar organization indicate a strong PEDOT $\leftrightarrow \mathrm{CNF}$ interaction in these samples as compared to the PEDOT $\leftrightarrow$ PEDOT interaction. The observations made are consistent with $\pi$-stacked PEDOT that covers the fibrils coaxially with a face-on orientation with respect to the surface of the fibrils. The TEM images which show stacks of crystals on the surface of the fibrils in the CNF-PEDOT:PSS samples are also in agreement with this model. The total surface area provided by the nano fibrils for PEDOT organization is several orders of magnitude larger than the surface area of the petri-dish substrate used to dry the self-standing films. Therefore, we conclude that the role of the petri-dish substrate in the PEDOT crystal formation is negligible. 
a) CNF-PEDOT:PSS

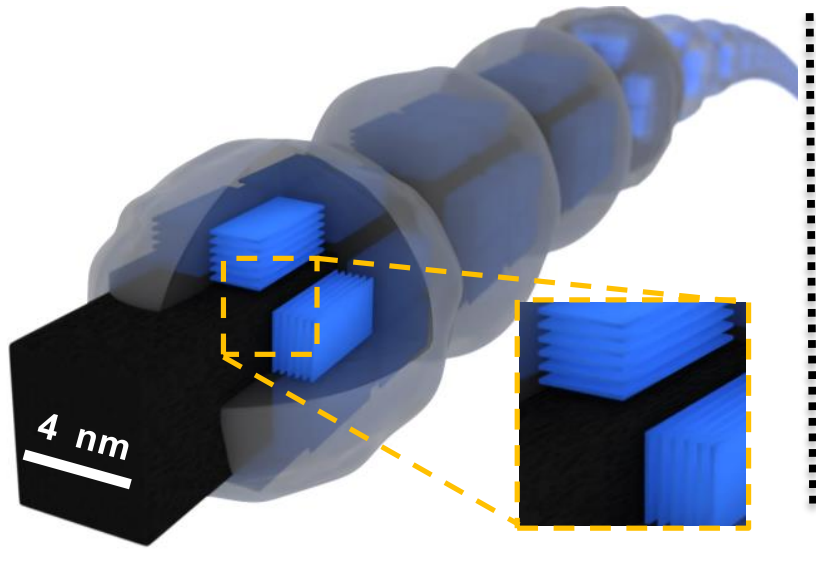

b) CMC-PEDOT:PSS

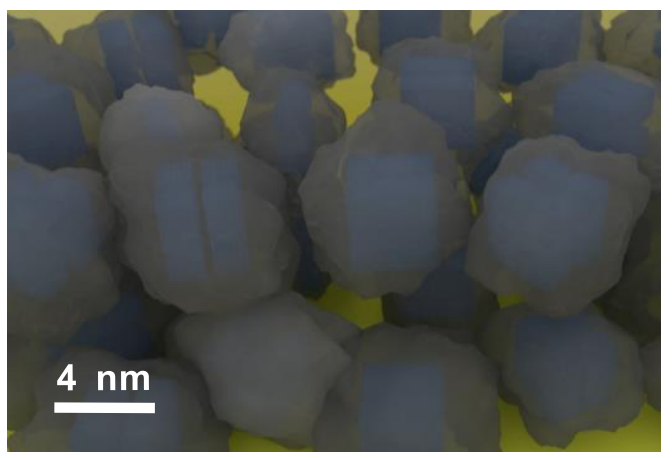

0.4

DS

Figure 6. Schematic model describing arrangement of PEDOT:PSS a) on CNF and b) with CMC

For the CMC-PEDOT:PSS samples, the surface available for the organization of the PEDOT crystals is the glass substrate onto which the film was cast. Hence, we expect the WAXS information to demonstrate the interaction of the PEDOT with the bottom of the free-standing film. The parallel incidence with the pronounced PEDOT lamellar peaks at the $90^{\circ}$ azimuthal angle (Fig. 4d) indicates a strong edge-on $\pi$-stacking with respect to the substrate level. On the other hand, the 010 peak at $90^{\circ}$ azimuthal angle indicates that there is also a considerable presence of $\pi$-stacked PEDOT in the face-on configuration. It is not clear why the PEDOT is found almost exclusively with edge-on and face-on orientations but not at other angles of orientation. Therefore, we speculate that the role of the substrate in the organization of the PEDOT extends throughout the bulk system.

PEDOT:PSS is seen to be highly malleable, forming conformal self-assembled structures on nanometer sized fibrils (Fig 6a). PEDOT:PSS, which has been described as a micelle, ${ }^{37}$ is in this study observed to form spherical vesicles on, or around, suitably sized nano-structures in the 
solution state. The nano-beads self-assemble on top of the fibrils in the wet state, as evident from the AFM sample preparation in which the PEDOT:PSS solution was drop cast on top of a predeposited CNF. This wet state aggregation of PEDOT is also in agreement with theoretical studies. ${ }^{59}$ As previously suggested, the wet state aggregation can be followed by the formation of highly defined $\pi$-stacked crystals of PEDOT during the evaporation of the solvents. ${ }^{37,43}$ In the systems with the polymeric CMC, PEDOT:PSS is phase segregated into micelle-like PEDOTrich structures embedded in a matrix of CMC and PSS.

Since the CNF-based and the CMC-based systems are markedly different from one another it is not trivial to construct a general theoretical model to describe their conductivity. However, by invoking available models for nano-particle conductors and two- and three-dimensional stick systems, we can qualitatively describe the observed difference in conductivity. The conductivity $\sigma$ of interconnected conducting nanoparticles is given by the equation $\sigma=\sigma_{0}\left(V_{f}-V_{c}\right)^{S}$ where $\sigma_{0}$ is the conductivity of a single nanoparticle, $V_{f}$ is the volumetric fraction of the nanoparticles, $V_{c}$ is the percolation volumetric fraction and $s$ is the critical exponent. The higher conductivity of the CNF-PEDOT:PSS correlates with a higher intrinsic conductivity of PEDOT nanoparticles due to improved $\pi$-stacking and lower percolation threshold due to high interconnectivity between nanoparticles on a single fibril. In addition, the theory of percolation thresholds in two- and threedimensional stick systems, ${ }^{60,61}$ states that the threshold percolation concentration $N_{c}$ of the nanosticks is proportional to $\frac{1}{L^{2} r}$ when $\mathrm{L} \gg \mathrm{r}$, where $\mathrm{L}$ and $\mathrm{r}$ are the length and radius of the nano-stick, respectively. If we assume that the $\mathrm{L}$ in the CNF based system is the length of the fibril while the $\mathrm{L}$ in the CMC system is the length of a single, phase segregated PEDOT-rich region (see Figure 3f), we can expect that the CNF system with the high-aspect ratio fibrils will lead to a lower percolation threshold as compared to the CMC system. We also note that single-fibril conductivity 
measurements would shed better light on whether it is possible to increase the conductivity of the CNF-PEDOT:PSS power paper in specific directions by an organized long-range alignment of the PEDOT:PSS coated CNFs in the paper.

Even though our study focused on cellulose:PEDOT:PSS systems, we note that similar nanoscale studies are indispensable for the full utilization of other bio-composite systems. This study shows the interaction between PEDOT:PSS and polymers or fibers. Hence, it is expected that other polymeric materials with similar structures will have similar properties to what is shown here, ${ }^{4}$ and it is possible that self-organizing effects are present in other previously investigated fibrillated systems. $^{5}$ Therefore, we believe that the results presented here are extendable to other related systems. Furthermore, the observation that CNF promotes a better organization of PEDOT:PSS as compared to $\mathrm{CMC}$ in the presence of a secondary dopant, here DMSO, shows that CNF too can be understood to act as an additional secondary dopant alongside DMSO. Therefore, our study extends the concept of secondary-doping from a solvent-based mechanism to other material systems, here nano-fibrils.

\section{CONCLUSIONS}

CNF proves to be a more versatile component in composite conducting papers, as compared to the common polymeric binder CMC. We have shown that, in addition to improving the mechanical stability, the nano-fibrils of CNF lead to high conductivity by virtue of the stronger surface interactions which create highly organized long-range conducting paths for charge transport. We used the direct imaging techniques AFM and TEM with specially adapted sample preparation steps to obtain images of PEDOT:PSS in a composite system with unprecedented clarity and resolution. These images confer a significant advance to our understanding of the nature of PEDOT:PSS crystallization. Furthermore, the structural organization was characterized with WAXS and 
consistent models of PEDOT assembly on cellulose have been suggested. We show that several properties of the cellulose-based additives, such as structural, dimensional and chemical compatibility, influence the resulting properties of the composite film. Despite the difficulties inherent in the characterization of blend systems, this work brings attention to the necessity of understanding the fundamental intra-component interactions in formulating state-of-the-art biocomposites for a sustainable future.

\section{METHODS}

Materials. CNF was prepared at RISE Bioeconomy from carboxymethylated softwood Kraft pulp by 10 passes through a high shear homogenizer at $700 \mathrm{MPa}$. Three different types of CNF with degrees of carboxymethyl substitution (DS) $0.06,0.12$, and 0.25 were prepared at $0.13 \mathrm{wt} \%$ in deionized water. The amount of charge was measured by conductometric titration. All CMCs (soluble non-fibrillated carboxymethylated cellulose) were obtained from Sigma Aldrich. The DS of the CMC was $0.76,0.85$, and 1.2. PEDOT:PSS with the product name PH $1000(1.1 \mathrm{wt} \%)$ was purchased from Clevios. Polyethyleneimine, DMSO and glycerol were obtained from Sigma Aldrich. Mica substrate for AFM was obtained from Caspilor.

Power paper. Conducting papers were prepared as described previously by Malti et al. ${ }^{8}$ In short the components cellulose: (PEDOT:PSS) : DMSO : glycerol were mixed in $1: 2: 11: 1.6$ dry weight ratio. For the CNF papers the solutions were mixed using a high shear mixer for $5 \mathrm{~min}$ while for the $\mathrm{CMC}$ the solutions were stirred with a magnetic stirrer. Each solution was poured on a petri dish and dried in an oven at $60^{\circ} \mathrm{C}$ resulting in a flexible conducting paper.

Electrical characterization. For the conductivity measurements, the free-standing conducting paper was cut in small pieces of $4 \times 20 \mathrm{~mm}$ and attached with conducting silver paste to a substrate 
with evaporated gold 4-probes. A Keithley model 2400 was used to apply a current across the outer 2 probes while simultaneously measuring the resistance between the inner 2 probes from which the conductivity was calculated. The thickness of the conducting paper was measured by the surface profiler Dektak3ST manufactured by Veeco.

Atomic force microscopy. AFM investigations were performed with a Dimension 3100 model Veeco microscope utilizing Nanoscope software for data acquisition in tapping-mode configuration giving topography and phase images. The CNF samples were investigated on mica substrate. The mica was cleaned by the scotch-tape technique ${ }^{55}$ and then a drop of $1 \mathrm{~g} / \mathrm{L}$ polyethylene imine (PEI) solution was put on it for $10 \mathrm{sec}$. The PEI solution was rinsed away with DI water and dried with $\mathrm{N}_{2}$ gas, leaving a layer of PEI to anchor the CNF. This was followed by dropping $0.043 \mathrm{wt} \%$ of CNF solution on top of the PEI monolayer for $20 \mathrm{sec}$. Then the solution was rinsed with DI water, dried with $\mathrm{N}_{2}$ gas and observed with AFM. For the PEDOT:PSS AFM samples, PH1000 was mixed with DMSO and glycerol at the ratio 1:0.02:0.005 (PH1000:DMSO:glycerol). A drop of this solution was also put for 50 seconds on top of the CNF lodged on mica and then rinsed off with DI water. The substrate was then heated on a hot plate at $100{ }^{\circ} \mathrm{C}$ for $5 \mathrm{~min}$ in order to remove excess solvents. CMC samples were prepared from a solution of CMC, PEDOT:PSS, DMSO and glycerol at the dry wt $\%$ ratio $1: 2: 11: 1.6$. This solution was spin casted at $1500 \mathrm{rpm}$ onto native-Si substrates cleaned by UV-ozone treatment. The substrates were then baked at $100^{\circ} \mathrm{C}$ for $5 \mathrm{~min}$.

Wide Angle X-ray Scattering. In order to make the self-standing films used in the WAXS experiments, PEDOT:PSS, DMSO, and glycerol were mixed in the ratio (1:0.8:5.5) and homogenized using a shear mixer for $5 \mathrm{~min}$. Following that the solution was dried in the oven at $70{ }^{\circ} \mathrm{C}$ giving free-standing films. WAXS measurements were done utilizing a $\mathrm{Cu} \mathrm{K}$-alpha X-ray 
source emitting at $1.5418 \AA$, monochromatized and focused by 1D multilayer optics. The X-rays scattered from the sample were collected in vacuum using a Fuji photo-stimulable imaging plate in transmission and grazing incidence. Further details of the WAXS instrumentation can be found in Apitz et al. ${ }^{56}$

TEM. Investigations were done on standard lacey carbon film suspended by copper TEM grids (obtained from SPI Supplies). HRTEM imaging was performed using the Linköping double Cs corrected FEI Titan3 60-300, operated at $300 \mathrm{kV}$. Pure CNF samples with DS 0.12 at 0.04 wt \% in water were dropped on the copper grid and rinsed with drops of water after 10 seconds. The copper grid was then placed on a hot plate at $100{ }^{\circ} \mathrm{C}$ for $3 \mathrm{~min} . \mathrm{CNF}$, PEDOT:PSS, DMSO were mixed at the dry wt $\%$ ratio $1: 8: 44$ at $0.04 \mathrm{wt} \%$ in water and dropped on the copper grid, followed by rinsing with water after 10 seconds. The copper grid was then placed on the hot plate at $100{ }^{\circ} \mathrm{C}$ for $3 \mathrm{~min}$.

\section{Competing interests}

The authors declare no competing financial interests.

Supporting Information: The following files are available free of charge.

Additional AFM/TEM/WAXS data and a theoretical model of PEDOT:PSS organization on CNF. (PEDOTPSSonCellulose_ACSAEM_SuppInfo_BelainehDetal.pdf)

\section{Corresponding Authors}

*(dagmawi.belaineh.yilma@ri.se)

*(isak.engquist@liu.se)

\section{Present Address}


${ }^{\dagger}$ Dagmawi Belaineh

RISE Research Institutes of Sweden

Division ICT

RISE Acreo

60117 Norrköping

Sweden

\section{Author Contributions}

The manuscript was written through contributions of all authors. All authors have given approval to the final version of the manuscript.

\section{Funding Sources}

Energimyndigheten (P43561-1), Stiftelsen för Strategisk Forskning.

\section{ACKNOWLEDGMENT}

We acknowledge A. Mehandzhiyski for the molecular dynamics simulations. We acknowledge

J. Gladisch for rendering the schematic models. We thank I. Zozoulenko, M. Modarresi, A. Fall, H. Francon, P. Larsson, and P. Persson for fruitful discussions.

\section{References}


(1) Zhu, H.; Luo, W.; Ciesielski, P. N.; Fang, Z.; Zhu, J.; Henriksson, G.; Himmel, M. E.; Hu, L. Wood-derived materials for green electronics, biological devices, and energy applications. Chemical reviews 2016, 116 (16), 9305-9374.

(2) Milczarek, G.; Inganäs, O. Renewable cathode materials from biopolymer/conjugated polymer interpenetrating networks. Science 2012, 335 (6075), 1468-1471.

(3) Pandey, J. K.; Takagi, H.; Nakagaito, A. N.; Saini, D. R.; Ahn, S.-H. An overview on the cellulose based conducting composites. Composites Part B: Engineering 2012, 43 (7), 2822-2826.

(4) Khan, S.; Narula, A. K. Bio-hybrid blended transparent and conductive films PEDOT: PSS: Chitosan exhibiting electro-active and antibacterial properties. European Polymer Journal 2016, 81, 161-172.

(5) Pal, R. K.; Farghaly, A. A.; Collinson, M. M.; Kundu, S. C.; Yadavalli, V. K. Photolithographic micropatterning of conducting polymers on flexible silk matrices. Advanced Materials 2016, 28 (7), 1406-1412.

(6) Zheng, G.; Cui, Y.; Karabulut, E.; Wågberg, L.; Zhu, H.; Hu, L. Nanostructured paper for flexible energy and electronic devices. MRS bulletin 2013, 38 (4), 320-325.

(7) Edberg, J.; Inganäs, O.; Engquist, I.; Berggren, M. Boosting the capacity of all-organic paper supercapacitors using wood derivatives. Journal of Materials Chemistry A 2018, 6 (1), 145-152.

(8) Abdellah, M.; Jesper, E.; Hjalmar, G.; Ullah, K. Z.; W., A. J.; Xianjie, L.; Dan, Z.; Hao, Z.; Yulong, Y.; W., B. J.; Isak, E.; Mats, F.; Lars, W.; Xavier, C.; Magnus, B. An Organic Mixed IonElectron Conductor for Power Electronics. Advanced Science 2016, 3 (2), 1500305.

(9) Luong, N. D.; Korhonen, J. T.; Soininen, A. J.; Ruokolainen, J.; Johansson, L.-S.; Seppälä, J. Processable polyaniline suspensions through in situ polymerization onto nanocellulose. European Polymer Journal 2013, 49 (2), 335-344.

(10) Shi, Z.; Phillips, G. O.; Yang, G. Nanocellulose electroconductive composites. Nanoscale 2013, 5 (8), 3194-3201.

(11) Dufresne, A. Cellulose-based composites and nanocomposites. In Monomers, polymers and composites from renewable resources; Elsevier: 2008; pp 401-418.

(12) Belgacem, M. N.; Gandini, A. Natural fibre-surface modification and characterization. Natural Fibre Reinforced Polymer Composites: From Macro to Nanoscale 2009, 14-46.

(13) Khalil, H. A.; Davoudpour, Y.; Islam, M. N.; Mustapha, A.; Sudesh, K.; Dungani, R.; Jawaid, M. Production and modification of nanofibrillated cellulose using various mechanical processes: a review. Carbohydrate polymers 2014, 99, 649-665.

(14) Czaja, W. K.; Young, D. J.; Kawecki, M.; Brown, R. M. The future prospects of microbial cellulose in biomedical applications. Biomacromolecules 2007, 8 (1), 1-12.

(15) Nordenström, M.; Fall, A.; Nystrom, G.; Wagberg, L. Formation of Colloidal Nanocellulose Glasses and Gels. Langmuir 2017, 33 (38), 9772-9780.

(16) Henriksson, M.; Henriksson, G.; Berglund, L.; Lindström, T. An environmentally friendly method for enzyme-assisted preparation of microfibrillated cellulose (MFC) nanofibers. European Polymer Journal 2007, 43 (8), 3434-3441.

(17) Berglund, L. A.; Peijs, T. Cellulose biocomposites from bulk moldings to nanostructured systems. MRS bulletin 2010, 35 (3), 201-207.

(18) Saito, T.; Hirota, M.; Tamura, N.; Kimura, S.; Fukuzumi, H.; Heux, L.; Isogai, A. Individualization of nano-sized plant cellulose fibrils by direct surface carboxylation using TEMPO catalyst under neutral conditions. Biomacromolecules 2009, 10 (7), 1992-1996.

(19) Thomas, H. New ionic polymers by cellulose functionalization. Macromolecular Chemistry and Physics 1998, 199 (11), 2341-2364. 
(20) Chou, S.-L.; Pan, Y.; Wang, J.-Z.; Liu, H.-K.; Dou, S.-X. Small things make a big difference: binder effects on the performance of Li and $\mathrm{Na}$ batteries. Physical Chemistry Chemical Physics 2014, 16 (38), 20347-20359.

(21) Elschner, A.; Kirchmeyer, S.; Lovenich, W.; Merker, U.; Reuter, K. PEDOT: principles and applications of an intrinsically conductive polymer, CRC Press: 2010.

(22) Groenendaal, L.; Jonas, F.; Freitag, D.; Pielartzik, H.; Reynolds, J. R. Poly (3, 4ethylenedioxythiophene) and its derivatives: past, present, and future. Advanced materials 2000, 12 (7), 481-494.

(23) Wang, G.; Zhang, L.; Zhang, J. A review of electrode materials for electrochemical supercapacitors. Chemical Society Reviews 2012, 41 (2), 797-828.

(24) Belaineh, D.; Png, R.-Q.; McGuiness, C. L.; Mathai, M.; Seshadri, V.; Ho, P. K. A highperformance p-doped conducting polymer blend based on sulfonated polyalkoxythiophene and poly (4-hydroxystyrene). Chemistry of Materials 2014, 26 (16), 4724-4730.

(25) Kirchmeyer, S.; Reuter, K. Scientific importance, properties and growing applications of poly(3,4-ethylenedioxythiophene). Journal of Materials Chemistry 2005, 15 (21), 2077-2088.

(26) Nilsson, D.; Chen, M.; Kugler, T.; Remonen, T.; Armgarth, M.; Berggren, M. Bi-stable and dynamic current modulation in electrochemical organic transistors. Advanced Materials 2002, 14 (1), 51-54.

(27) Yue, R.; Xu, J. Poly(3,4-ethylenedioxythiophene) as promising organic thermoelectric materials: A mini-review. Synthetic Metals 2012, 162 (11), 912-917.

(28) Zhang, M.; Yuan, W.; Yao, B.; Li, C.; Shi, G. Solution-Processed PEDOT:PSS/Graphene Composites as the Electrocatalyst for Oxygen Reduction Reaction. ACS Applied Materials \& Interfaces 2014, 6 (5), 3587-3593.

(29) Feng-Xing, J.; Jing-Kun, X.; Bao-Yang, L.; Yu, X.; Rong-Jin, H.; Lai-Feng, L. Thermoelectric performance of poly (3, 4-ethylenedioxythiophene): poly (styrenesulfonate). Chinese Physics Letters 2008, 25 (6), 2202.

(30) Hou, Y.; Cheng, Y.; Hobson, T.; Liu, J. Design and Synthesis of Hierarchical MnO2 Nanospheres/Carbon Nanotubes/Conducting Polymer Ternary Composite for High Performance Electrochemical Electrodes. Nano Letters 2010, 10 (7), 2727-2733.

(31) Nardes, A. M.; Kemerink, M.; Janssen, R. A.; Bastiaansen, J. A.; Kiggen, N. M.; Langeveld, B. M.; Van Breemen, A. J.; De Kok, M. M. Microscopic understanding of the anisotropic conductivity of PEDOT: PSS thin films. Advanced Materials 2007, 19 (9), 1196-1200.

(32) Palumbiny, C. M.; Liu, F.; Russell, T. P.; Hexemer, A.; Wang, C.; Müller-Buschbaum, P. The crystallization of PEDOT: PSS polymeric electrodes probed in situ during printing. Advanced Materials 2015, 27 (22), 3391-3397.

(33) Crispin, X.; Marciniak, S.; Osikowicz, W.; Zotti, G.; Van Der Gon, A. D.; Louwet, F.; Fahlman, M.; Groenendaal, L.; De Schryver, F.; Salaneck, W. R. Conductivity, morphology, interfacial chemistry, and stability of poly (3, 4-ethylene dioxythiophene)-poly (styrene sulfonate): A photoelectron spectroscopy study. Journal of polymer science Part B: Polymer physics 2003, 41 (21), 2561-2583.

(34) Xia, Y.; Ouyang, J. Significant different conductivities of the two grades of poly (3, 4ethylenedioxythiophene): poly (styrenesulfonate), Clevios P and Clevios Ph1000, arising from different molecular weights. ACS applied materials \& interfaces 2012, 4 (8), 4131-4140.

(35) Wang, G.-F.; Tao, X.-M.; Xin, J. H.; Fei, B. Modification of conductive polymer for polymeric anodes of flexible organic light-emitting diodes. Nanoscale research letters 2009, 4 (7), 613. 
(36) Volkov, A. V.; Wijeratne, K.; Mitraka, E.; Ail, U.; Zhao, D.; Tybrandt, K.; Andreasen, J. W.; Berggren, M.; Crispin, X.; Zozoulenko, I. V. Understanding the capacitance of PEDOT: PSS. Advanced Functional Materials 2017, 27 (28), 1700329.

(37) Takano, T.; Masunaga, H.; Fujiwara, A.; Okuzaki, H.; Sasaki, T. PEDOT nanocrystal in highly conductive PEDOT: PSS polymer films. Macromolecules 2012, 45 (9), 3859-3865.

(38) Timpanaro, S.; Kemerink, M.; Touwslager, F.; De Kok, M.; Schrader, S. Morphology and conductivity of PEDOT/PSS films studied by scanning-tunneling microscopy. Chemical Physics Letters 2004, 394 (4-6), 339-343.

(39) Ouyang, J.; Xu, Q.; Chu, C.-W.; Yang, Y.; Li, G.; Shinar, J. On the mechanism of conductivity enhancement in poly(3,4-ethylenedioxythiophene):poly(styrene sulfonate) film through solvent treatment. Polymer 2004, 45 (25), 8443-8450.

(40) Alemu, D.; Wei, H.-Y.; Ho, K.-C.; Chu, C.-W. Highly conductive PEDOT: PSS electrode by simple film treatment with methanol for ITO-free polymer solar cells. Energy \& environmental science 2012, 5 (11), 9662-9671.

(41) Kim, N.; Lee, B. H.; Choi, D.; Kim, G.; Kim, H.; Kim, J.-R.; Lee, J.; Kahng, Y. H.; Lee, K. Role of interchain coupling in the metallic state of conducting polymers. Physical review letters 2012, 109 (10), 106405.

(42) Wang, X.; Liu, P.; Jiang, Q.; Zhou, W.; Xu, J.; Liu, J.; Jia, Y.; Duan, X.; Liu, Y.; Du, Y. Efficient DMSO-Vapor Annealing for Enhancing Thermoelectric Performance of PEDOT: PSSBased Aerogel. ACS applied materials \& interfaces 2018, 11 (2), 2408-2417.

(43) Franco-Gonzalez, J. F.; Zozoulenko, I. V. Molecular Dynamics Study of Morphology of Doped PEDOT: From Solution to Dry Phase. The Journal of Physical Chemistry B 2017, 121 (16), 4299-4307.

(44) Montibon, E.; Järnström, L.; Lestelius, M. Characterization of poly (3, 4ethylenedioxythiophene)/poly (styrene sulfonate)(PEDOT: PSS) adsorption on cellulosic materials. Cellulose 2009, 16 (5), 807-815.

(45) Gangopadhyay, R.; De, A. Conducting polymer nanocomposites: a brief overview. Chemistry of materials 2000, 12 (3), 608-622.

(46) Hammond, P. T. Form and function in multilayer assembly: new applications at the nanoscale. Advanced Materials 2004, 16 (15), 1271-1293.

(47) Nyholm, L.; Nyström, G.; Mihranyan, A.; Strømme, M. Toward flexible polymer and paperbased energy storage devices. Advanced Materials 2011, 23 (33), 3751-3769.

(48) Zhang, L.; Du, W.; Nautiyal, A.; Liu, Z.; Zhang, X. Recent progress on nanostructured conducting polymers and composites: synthesis, application and future aspects. Science China Materials 2018, 1-50.

(49) Shi, H.; Liu, C.; Jiang, Q.; Xu, J. Effective approaches to improve the electrical conductivity of PEDOT: PSS: a review. Advanced Electronic Materials 2015, 1 (4), 1500017.

(50) Stöcker, T.; Köhler, A.; Moos, R. Why does the electrical conductivity in PEDOT: PSS decrease with PSS content? A study combining thermoelectric measurements with impedance spectroscopy. Journal of polymer science part B: polymer physics 2012, 50 (14), 976-983.

(51) Chang, S. H.; Chen, C.-C.; Cheng, H.-M.; Chen, S.-H. Structural, Optical, Electrical and Electronic Properties of PEDOT: PSS Thin Films and Their Application in Solar Cells. Printable Solar Cells 2017, 263-288.

(52) Aleshin, A. N.; Williams, S. R.; Heeger, A. J. Transport properties of poly(3,4ethylenedioxythiophene)/poly(styrenesulfonate). Synthetic Metals 1998, 94 (2), 173-177. 
(53) Ionescu-Zanetti, C.; Mechler, A.; Carter, S. A.; Lal, R. Semiconductive polymer blends: correlating structure with transport properties at the nanoscale. Advanced Materials 2004, 16 (5), 385-389.

(54) Nardes, A. M.; Janssen, R. A.; Kemerink, M. A morphological model for the solventenhanced conductivity of PEDOT: PSS thin films. Advanced Functional Materials 2008, 18 (6), 865-871.

(55) Crispin, X.; Jakobsson, F.; Crispin, A.; Grim, P.; Andersson, P.; Volodin, A.; Van Haesendonck, C.; Van der Auweraer, M.; Salaneck, W. R.; Berggren, M. The origin of the high conductivity of poly (3, 4-ethylenedioxythiophene)- poly (styrenesulfonate)(PEDOT- PSS) plastic electrodes. Chemistry of Materials 2006, 18 (18), 4354-4360.

(56) Wang, Y.; Song, R.; Li, Y.; Shen, J. Understanding tapping-mode atomic force microscopy data on the surface of soft block copolymers. Surface science 2003, 530 (3), 136-148.

(57) Rivnay, J.; Mannsfeld, S. C.; Miller, C. E.; Salleo, A.; Toney, M. F. Quantitative determination of organic semiconductor microstructure from the molecular to device scale. Chemical reviews 2012, 112 (10), 5488-5519.

(58) Mehandzhiyski, A. Y.; Zozoulenko, I. Computational Microscopy of PEDOT:PSS/Cellulose Composite Paper. ACS Applied Energy Materials 2019.

(59) Franco-Gonzalez, J. F.; Rolland, N.; Zozoulenko, I. V. Substrate-Dependent Morphology and Its Effect on Electrical Mobility of Doped Poly (3, 4-ethylenedioxythiophene)(PEDOT) Thin Films. ACS applied materials \& interfaces 2018, 10 (34), 29115-29126.

(60) Balberg, I.; Binenbaum, N. Computer study of the percolation threshold in a two-dimensional anisotropic system of conducting sticks. Physical Review B 1983, 28 (7), 3799.

(61) Balberg, I.; Binenbaum, N.; Wagner, N. Percolation thresholds in the three-dimensional sticks system. Physical Review Letters 1984, 52 (17), 1465. 